\title{
Report of the American Heart Association (AHA) Scientific Sessions 2014, Chicago
}

\author{
Takashi Kohno, MD, PhD
}

\begin{abstract}
The American Heart Association (AHA) Scientific Sessions were held in Chicago on November 15-19, 2014. The meeting attracted more than 17,000 participants, including physicians, research scientists, students, and paramedical personnel, from more than 100 countries. Sessions over the 5 days included comprehensive and unparalleled education delivered via more than 5,000 presentations, with 1,000 invited faculty members and 4,000 abstract presentations from world leaders in cardiovascular (CV) disease. There were 16 trials scheduled in 4 late-breaking clinical trial sessions. The Dual Antiplatelet Therapy study revealed that aspirin plus a thienopyridine beyond 1 year subsequent to placement of a drug-eluting stent, as compared with aspirin therapy alone, significantly reduced stent thrombosis and major CV and cerebrovascular events but was associated with increased risk of bleeding. The IMPROVE-IT research showed that, relative to simvastatin with placebo, simvastatin with $10 \mathrm{mg}$ of ezetimibe daily led to a significantly lower primary combined endpoint in moderate- to high-risk patients, who stabilized following acute coronary syndrome. This was the first trial to demonstrate incremental clinical benefit by adding a nonstatin agent to statin therapy and reaffirmed the low-density lipoprotein (LDL) hypothesis stating that reducing LDL-cholesterol prevents CV events. Summaries and overviews of both the late-breaking trials and the sessions to which members of the Japanese Circulation Society contributed are presented. (Circ J 2015; 79: 34-40)
\end{abstract}

Key Words: American Heart Association; Japanese Circulation Society; Late-breaking clinical trials; Scientific Sessions

$\mathbf{T}$ he American Heart Association (AHA) Scientific Sessions 2014 were held on November 15-19, 2014 at McCormick Place in Chicago (Figures 1A-D), the city in which the AHA was founded 90 years ago at the Drake Hotel by 6 cardiologists. The AHA Scientific Sessions encompass the best science and are one of the leading cardiovascular (CV) conferences for basic, translational, clinical, and population science in the United States, ${ }^{1-3}$ with more than 17,000 professional attendees, including many Japanese physicians and researchers. Sessions include comprehensive, unparalleled education delivered via more than 5,000 presentations, with 1,000 invited lectures and 4,000 abstracts of original research presentations. The main program included 4 late-breaking clinical trial sessions consisting of presentation of 16 clinical studies; 5 clinical science; special reports sessions; 9 plenary sessions; and 18 special sessions. This year, almost $90 \%$ of the abstracts accepted were presented as posters to facilitate discussion, engagement, and networking. The e-Abstract sessions were presented in theaters featured in each core poster community, highlighting the must-see science of the day. Moderators led lively discussions between presenters and the audience (Figure 2A). This paper reports the highlights and several key presentations from AHA Scientific Sessions 2014.

\section{Opening Session}

In the opening session, the AHA President, Professor Elliott Antman, stated that scientific researchers and healthcare professionals must transform emerging technology and big data into innovative ways of helping patients (Figure 2B). He urged increased innovation in the fight against heart disease and stroke, which are the leading causes of death worldwide. $\mathrm{He}$ also emphasized the fact that new diagnostic and therapeutic options are being discovered at a pace previously unseen in human history, and we have an unprecedented opportunity to harness these advances to save and improve lives. As an example of a new diagnostic option, he cited a heart-rhythm monitoring device. Using this device, the patient could take readings using an attachment to a smart phone case and email the results to cardiologists. He also introduced results of a study, published in 2013, examining the effect of the anticoagulant, edoxaban, on stroke prevention, in addition to a larger analysis of the effectiveness of 3 newer oral anticoagulants. ${ }^{4} \mathrm{He}$ detailed the history of such drugs to show that innovation is required. He also referenced the first funded researcher in the ground-breaking Cardiovascular GenomePhenome Study, which is built on the big data of numerous

The opinions expressed in this article are not necessarily those of the editors or of the Japanese Circulation Society.

Received December 3, 2014; accepted December 4, 2014; released online December 15, 2014

Division of Cardiology, Department of Medicine, Keio University School of Medicine, Tokyo, Japan

Mailing address: Takashi Kohno, MD, PhD, Division of Cardiology, Department of Medicine, Keio University School of Medicine, 35

Shinanomachi, Shinjuku-ku, Tokyo 160-8582, Japan. E-mail: kohno@a2.keio.jp

ISSN-1346-9843 doi:10.1253/circj.CJ-14-1329

All rights are reserved to the Japanese Circulation Society. For permissions, please e-mail: cj@j-circ.or.jp 

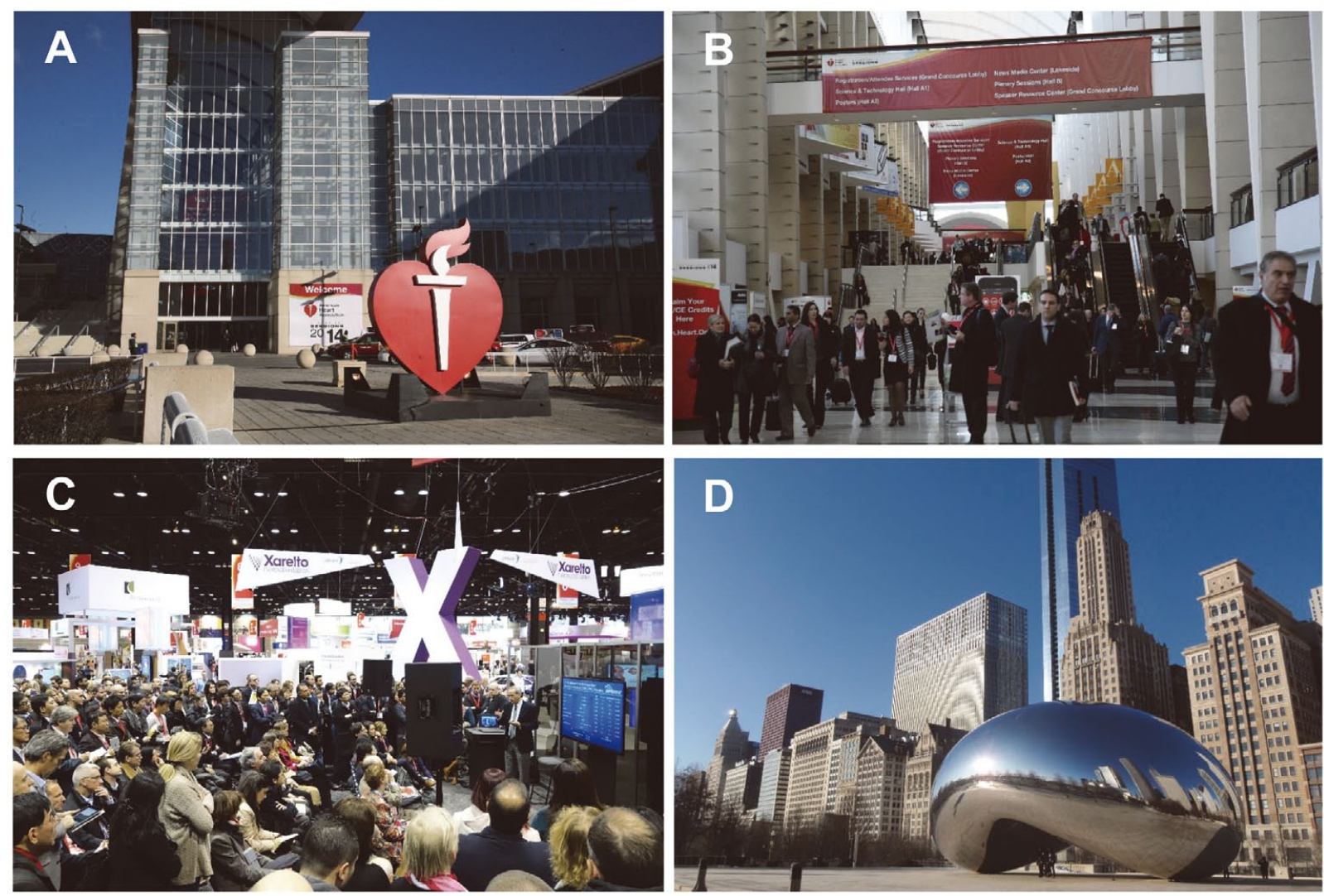

Figure 1. The American Heart Association Scientific Sessions 2014 were held at McCormick Place (A: Main Entrance, B: Grand Concourse Lobby, C: Cardiovascular Expert Theater) in Chicago (D).
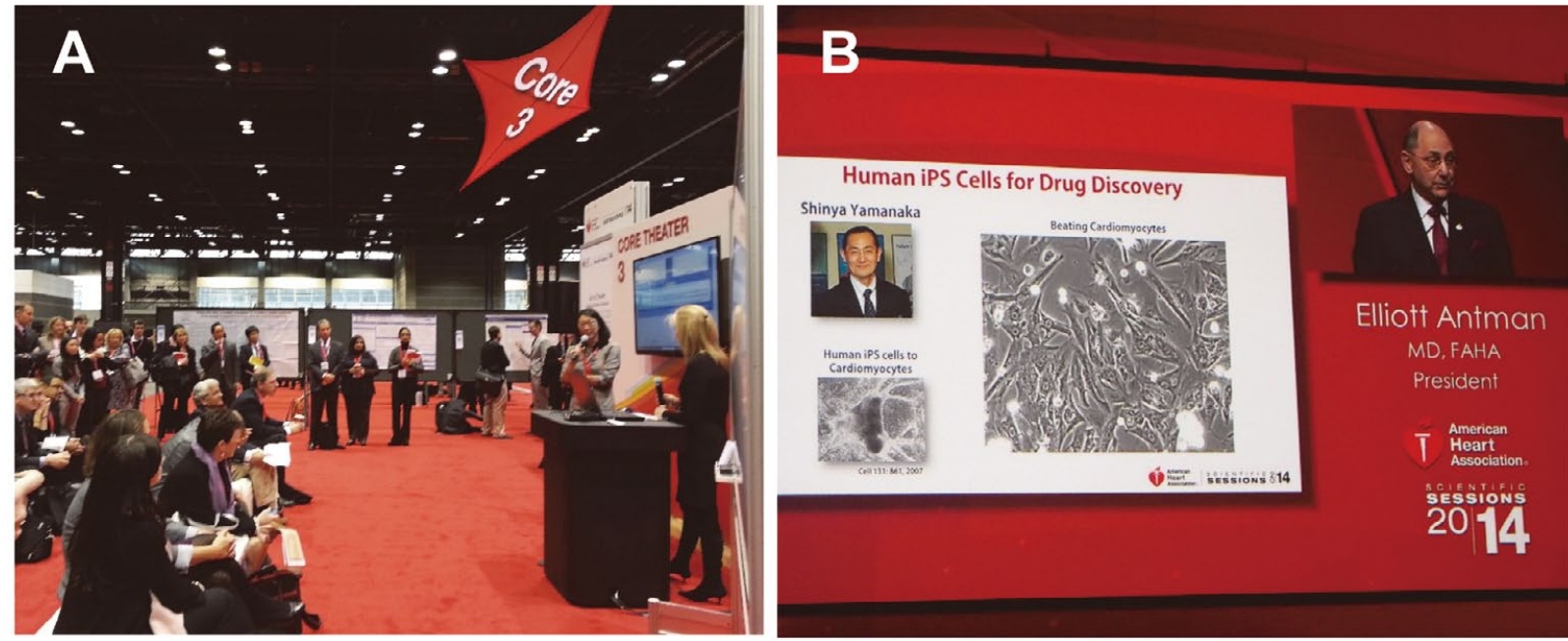

Figure 2. The latest basic and clinical sciences presented in poster sessions, with presenters and audiences participating actively in discussions (A). American Heart Association President, Dr Elliott Antman, in his opening session, emphasized that scientific researchers must transform emerging technology and big data into innovative ways of helping patients (B). 

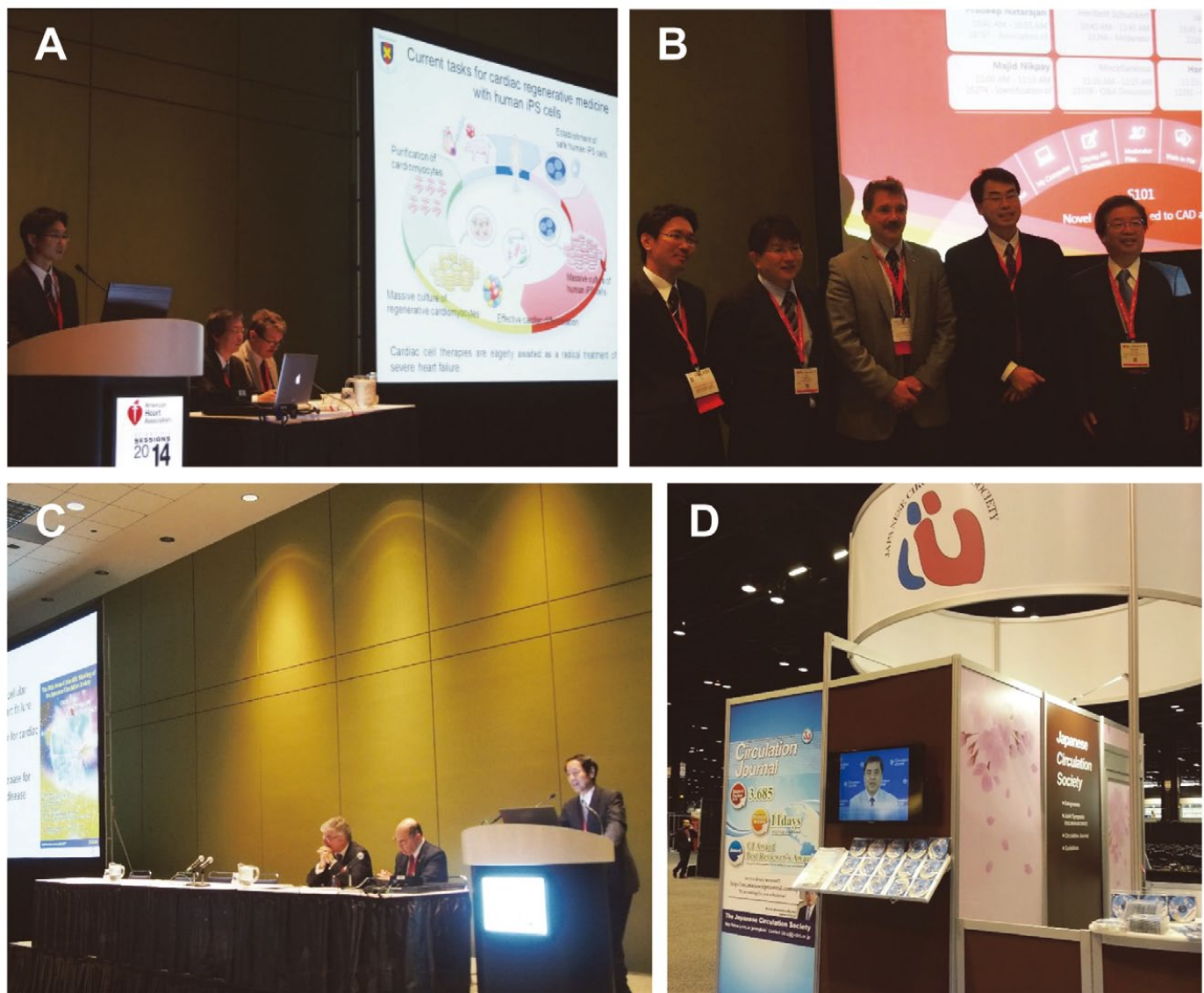

Figure 3. In the AHA-JCS Joint Session organized by Professors K Fukuda and CE Murry, "Use of ES/iPS cells for cardiovascular medicine" was discussed (A). From left to right, Dr Jun Fujita, Professors Jun Yamashita, Charles E Murry (Chair), Joseph Wu, and Keiichi Fukuda (Chair) (B). The AHA Scientific Sessions 2014 acknowledged the major international societies for their contribution to the International Sessions. Professor Nagai presented JCS activity in the Best of Other Cardiovascular Meetings (C). The JCS also manned a booth in the Science and Technology Hall (D).

studies, including the Framingham Heart Study and the Jackson Heart Study.

\section{The Japanese Circulation Society (JCS) in the AHA Scientific Sessions}

The JCS maintains a close relationship with the AHA. This year, the AHA-JCS Joint Session's theme was the use of embryonic stem (ES)/induced pluripotent stem (iPS) cells for CV medicine. Professors Keiichi Fukuda, from Keio University, and Charles E Murry, from University of Washington, organized this session (Figures 3A,B). The representative researchers for ES/iPS cells from the JCS and the AHA discussed current topics. Dr Jun Fujita, from Keio University, emphasized the establishment of cell culture systems for large numbers of regenerative cardiomyocytes and safe transplantation as keys to cardiac regenerative medicine..$^{5}$ Professor Jun Yamashita, from Kyoto University, presented longer engraftment of transplanted iPS cells, which will open the new frontier of regenerative therapies with iPS cells for patients with severe heart failure (HF). Professor William Pu talked about the disease model with patient-derived iPS cells. Finally, Professor Joseph Wu summarized the utility and tasks of iPS cell-derived cardiomyocytes. There were numerous people in the audience for this session, suggesting that regenerative therapy with human pluripotent stem cells attracts increased attention from cardiology researchers.

The AHA 2014 acknowledged major international societies, including the JCS, for their contributions to the international sessions. In the Best of Other Cardiovascular Meetings (international), Professor Ryozo Nagai, Congress Chairperson of the 78th Annual Scientific Meeting of the JCS, reviewed the meeting held in Tokyo between March 21 and 23, 2014, in which the main theme was "Cardiology in the information era: from bench to community" (Figure 3C). He also demonstrated the effect of Krüppel-like factors in $\mathrm{CV}$ remodeling ${ }^{6}$ and emphasized the role of heterocellular interplay in HF. The JCS also featured a booth in the Science and Technology Hall to advertise JCS activities to AHA attendees (Figure 3D).

\section{Late-Breaking Clinical Trials}

Updates of results from the most recent clinical trials were the highlight of the AHA Scientific Sessions, with 16 late-breaking clinical trials presented (Figure 4A). 

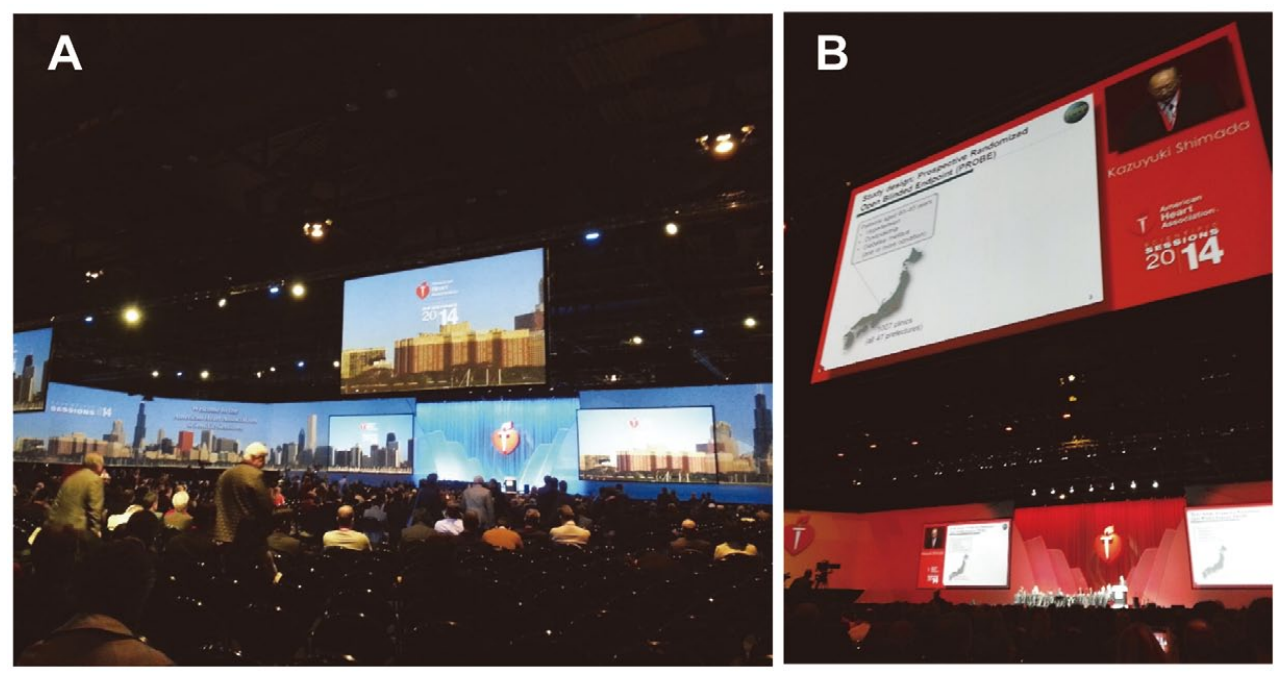

Figure 4. Updated results of clinical trials were reported in the Late-Breaking Clinical Trials Sessions (A). Dr Kazuyuki Shimada, Shin-Oyama City Hospital, presenting the Japanese Primary Prevention Project (JPPP) in the late-breaking clinical trials (B).

\section{Antiplatelet Therapies Following Coronary Stenting The Dual Antiplatelet Therapy (DAPT) Study Extending} the use of aspirin plus a thienopyridine beyond 1 year subsequent to placement of a drug-eluting stent (DES), as compared with aspirin therapy alone, significantly reduced the rate of stent thrombosis (ST) and major CV and cerebrovascular events but was associated with an increased risk of bleeding. ${ }^{7}$ This international study evaluated 22,866 patients who had received DES. All received aspirin therapy plus a thienopyridine drug (clopidogrel or prasugrel). After 12 months, 9,961 of those who had tolerated DAPT were randomly assigned to continue receiving thienopyridine treatment or a placebo for a further 18 months; all patients continued receiving aspirin. Continued treatment with thienopyridine, relative to a placebo, reduced rates of ST $(0.4 \%$ vs. $1.4 \% ; \mathrm{P}<0.001)$ and major adverse CV and cerebrovascular events (a composite of death, myocardial infarction $[\mathrm{MI}]$, or stroke; $4.3 \%$ vs. $5.9 \%$; $\mathrm{P}<0.001$ ). The rate of moderate to severe bleeding increased with continued thienopyridine treatment $(2.5 \%$ vs. $1.6 \% ; \mathrm{P}=0.001)$, although fatal bleeding was rare in both groups. The continued thienopyridine treatment did not lower the rates of stroke and death. Prevention of MI and ST with continued thienopyridine treatment was consistent across patient groups, drugs, and stent types. The key message of this study was not only that some patients treated with a DES may benefit from extended DAPT, but also that the potential harm with this approach should not be overlooked. Presenting on behalf of the DAPT investigators, Dr Laura Mauri, an interventional cardiologist at Brigham and Women's Hospital, posited that physicians should consider individual patient risks when prescribing DAPT, particularly as the trial excluded patients with a history of major bleeding, either prior to the stent-placement procedure or within the first year of treatment. Further, we should be mindful that the applicability of this conclusion to Japanese patients, for whom late ST is much less common, ${ }^{8}$ remains uncertain, and a randomized controlled trial involving Japanese participants would be required to confirm the benefits and risks of DAPT beyond 1 year subsequent to placement of a DES in this population.
TAXUS Libertē Post Approval Study (TL-PAS) Prasugrel and aspirin administration was continued for 30 months and reduced the number of ischemic events for patients with the TAXUS Libertē paclitaxel-eluting stent, via reductions in MI and ST. ${ }^{9}$ The TL-PAS contributed to the DAPT study by randomizing patients receiving TAXUS Libertē stent to blinded prasugrel or matched placebo for 12-30 months following stent implantation. Withdrawal of prasugrel treatment after 12 months was associated with significantly higher incidence of major coprimary composite endpoints (all-cause death, MI, or stroke; $8.8 \%$ vs. $3.7 \%$; $\mathrm{P}<0.001$ ) relative to extended use of prasugrel. MI increased significantly with withdrawal of prasugrel ( $7.1 \%$ vs. $1.9 \% ; \mathrm{P}<0.001)$, and a difference in MI rates was found as early as 90 days subsequent to prasugrel withdrawal. The number of major bleeding events did not differ significantly between the prolonged treatment group and other group $(2.4 \%$ vs. $1.7 \%, \mathrm{P}=0.234)$.

ISAR-SAFE and ITALIC With guidelines recommending 12-month DAPT following stenting, European cardiologists typically advocate a shorter course of therapy to reduce the risk of associated bleeding. The ISAR-SAFE trial compared DAPT with clopidogrel and aspirin at 6 and 12 months to address the question of optimal duration of DAPT with clopidogrel. The trial was stopped after 4,005 patients had been included, because of the slow recruitment rate and low number of events; 6 months demonstrated non-inferiority over 12 months' administration of clopidogrel with respect to the primary composite endpoint (death, MI, ST, stroke, or major bleeding).

The ITALIC trial was a multicenter, randomized study involving patients undergoing implantation of Xience $\mathrm{V}$ and receiving either 6- or 24-month DAPT with confirmed nonresistance to aspirin. There was no significant difference between the 6- and 24- month DAPT groups with respect to the primary composite endpoint (all-cause death, MI, urgent target revascularization, stroke, or major bleeding at 12 months post stenting; $1.6 \%$ vs. $1.5 \%, \mathrm{P}=0.85$ ), even in high-risk (acute coronary syndrome $[\mathrm{ACS}]$ ) patients. There were no significant differences in ST or rates of bleeding complications between groups. 


\section{Summary of 4 Trials Regarding DAPT Therapy}

Professor Montalescot from the University of Paris VI provided much-needed context to the 4 trials, which appeared to contradict each other. The trials highlighting DAPT usage beyond 12 months showed lower rates of MI, ST, and death but more bleeding. In contrast, those advocating DAPT for less than 12 months showed similar rates for early ST, MI, and death but less bleeding. These findings suggest that a shorter course of therapy is safer, but a longer course of therapy prevents secondary events such as MI. For patients at risk of bleeding (eg, prior bleeding episodes, advanced age, need for surgery or anticoagulation therapy, or comorbidities associated with risk), DAPT during the 6 months following stent placement may be sufficient. For patients at risk of ischemic events (eg, patients with coronary artery disease (CAD), first-generation stents, or prior ST), extended DAPT beyond 12 months could be advocated.

\section{Anti-Lipid Therapy and Prevention of CAD}

IMPROVE-IT The IMPROVE-IT (Improved Reduction of Outcomes: Vytorin Efficacy International Trial) research compared 2 approaches: simvastatin with a placebo or simvastatin with ezetimibe. Both of the latter drugs reduce low-density lipoprotein cholesterol (LDL-C) but in different ways: simvastatin blocks the cholesterol produced by the liver, whereas ezetimibe reduces cholesterol absorption through inhibition of Niemann-Pick $\mathrm{C}_{1}$-like 1 protein. This study was a multicenter, randomized, double-blind trial involving 18,144 moderate-tohigh-risk patients, who were stabilized following ACS. Relative to simvastatin with a placebo, simvastatin taken with $10 \mathrm{mg}$ of ezetimibe daily reduced ischemic stroke by $21 \%$ and MI by $13 \%$ and led to a significantly lower incidence of the primary combined endpoint (CV death, MI, rehospitalization for unstable angina, coronary revascularization, or stroke; $34.7 \%$ vs. $32.7 \%, \mathrm{P}=0.016$ ). Mean LDL-C was significantly lower in patients treated with simvastatin and ezetimibe relative to those treated with simvastatin and a placebo $(53 \mathrm{mg} / \mathrm{dl}$ vs. $70 \mathrm{mg} / \mathrm{dl}$ at 1 year). This was the first trial to demonstrate incremental clinical benefit by adding a nonstatin agent to statin therapy and reaffirmed the LDL hypothesis stating that reducing LDL-C prevents CV events.

ODYSSEY ALTERNATIVE This study showed that alirocum$\mathrm{ab}$, a proprotein convertase subtilisin/kexin type-9 (PCSK9) inhibitor, provided significantly superior lipid reduction in statin-intolerant patients with high LDL-C levels relative to ezetimibe. The 314 patients were randomized to receive alirocumab as a $75-\mathrm{mg}$ self-administered injection fortnightly $(\mathrm{n}=126), 10 \mathrm{mg}$ ezetimibe daily $(\mathrm{n}=125)$, or $20 \mathrm{mg}$ atorvastatin daily $(n=63)$ for 24 weeks. The alirocumab dose was increased to $150 \mathrm{mg}$ at 12 weeks, depending on CV risk and LDL-C levels at 8 weeks. At 24 weeks, a mean reduction in LDL-C (primary endpoint) of $45 \%$ from baseline was observed in patients receiving alirocumab, with a $14.6 \%$ reduction observed in patients receiving ezetimibe.

Japanese Primary Prevention Project (JPPP) Low-dose aspirin, taken once daily, in elderly Japanese patients with atherosclerosis risk factors did not significantly prevent primary CV events relative to those observed in patients who received no aspirin. ${ }^{10}$ The JPPP was a multicenter, open-label, randomized, parallel group trial in which 14,658 patients aged 60-85 years with hypertension, high cholesterol, or diabetes were randomized to receive either $100 \mathrm{mg}$ enteric-coated aspirin per day $(n=7,323)$ or no aspirin $(n=7,335)$. Dr Kazuyuki Shimada from Shin-Oyama City Hospital reported that after 5 years there was no difference in composite primary outcome (death from CV causes [MI, stroke, and other CV causes], nonfatal stroke, or nonfatal MI; $2.8 \%$ for aspirin vs. $3.0 \%$ for no aspirin, $\mathrm{P}=0.54$ ) (Figure $4 \mathrm{~B}$ ).

FACTOR 64 Among asymptomatic patients with type 1 or 2 diabetes, screening for CAD with a coronary computed tomography angiography (CCTA) did not reduce CV events at 4 years, despite differential use of coronary interventions and favorable trends in lipids and blood pressure. ${ }^{11}$ The study involved 900 high-risk patients with diabetes from 45 clinics and practices within a single healthcare system, who were randomly assigned to receive CCTA $(n=452)$ or guideline-based optimal diabetes care $(n=447)$. The primary composite endpoint for all-cause mortality, non-fatal MI, or hospitalization for unstable angina did not differ significantly between the CCTA and control groups $(6.2 \%$ vs. $7.6 \%, \mathrm{P}=0.38)$. These findings do not support CCTA screening in this population.

\section{Structural Heart Disease}

Incidence of Infective Endocarditis in the UK In the United Kingdom, an increase in the incidence of infective endocarditis was temporally associated with the National Institute for Health and Care Excellence (NICE) 2008 recommendation that patients do not need to take antibiotics prior to invasive dental procedures or certain nondental procedures to prevent infective endocarditis. Antibiotic prescribing data showed that since 2008 , there has been an $88 \%$ reduction in prescriptions for amoxicillin and clindamycin (mean 10,900 prescriptions per month prior to March 2008 to 2,236 from March 2008, $\mathrm{P}<0.0001)$. Coinciding with the timing of the NICE guidelines, the number of cases of infective endocarditis increased significantly, above the projected historical trend, by 0.11 cases per 10 million people per month $(\mathrm{P}<0.0001)$. Based on this data, NICE issued a press release stating that, as a result of the data, it would undertake an immediate review of the guidance. Dr Mark J. Dayer, consultant cardiologist at the Taunton and Somerset NHS Trust, was cautious in the interpretation of the data and stated that a randomized clinical trial was required to determine whether there is a cause-effect relationship evident therein.

Role of Losartan in Marfan's Syndrome and Cardiomyopathy: the Pediatric Heart Network and INHERIT The Pediatric Heart Network conducted a randomized trial comparing losartan $(n=303)$ with atenolol $(n=305)$ in children and young adults with Marfan's syndrome. Losartan, an angiotensin II receptor antagonist, did not lead to a difference in the rate of aortic enlargement over a 3-year period relative to the commonly used drug, atenolol. No patient subgroups, defined according to age, $\beta$-blocker use, aortic root diameter at baseline, or sex, were observed to benefit from losartan. ${ }^{12}$

In the INHERIT study, losartan was evaluated in patients with hypertrophic cardiomyopathy (HCM). INHERIT was a single center, double-blind, placebo-controlled, randomized trial conducted in Denmark. Patients with HCM were randomized to $100 \mathrm{mg}$ losartan $(n=64)$ or a placebo $(n=69)$ daily for 12 months. Losartan was not shown to decrease left ventricular mass in patients with $\mathrm{HCM}(\mathrm{P}=0.60)$. In addition, there was no effect observed with respect to maximal wall thickness, left ventricular fibrosis, diastolic parameters, or exercise capacity. This study suggested that losartan may be used for other indications in patients with HCM, regardless of obstructive pathology.

Cardiothoracic Surgical Trials Network (CTSN) In patients with moderate ischemic mitral regurgitation, the addition of mitral valve repair (MVR) to coronary artery bypass grafting $(\mathrm{CABG})$ did not result in a higher degree of left ventricular 
reverse remodeling. ${ }^{13}$ The CTSN study randomized 301 patients with mitral regurgitation to $\mathrm{CABG}$ alone $(\mathrm{n}=151)$ or CABG+MVR ( $\mathrm{n}=150)$ to determine left ventricular remodeling, as measured by left ventricular end systolic volume index, using imaging. After 12 months, there was no difference in the primary endpoint of left ventricular end-systolic volume index between the treatment groups $(\mathrm{P}=0.61)$. The addition of MVR was associated with longer bypass time $(\mathrm{P}<0.001)$, longer postsurgical hospital stays $(\mathrm{P}=0.002)$, and a higher number of neurological events $(\mathrm{P}=0.03)$. Moderate or severe mitral regurgitation was less common in the combined-procedure group relative to the $\mathrm{CABG}$ group ( $11.2 \%$ vs. $31.0 \%, \mathrm{P}<0.001)$. Based on these data, the routine addition of MVR to CAGB for patients with moderate ischemic mitral regurgitation may not be warranted. Long-term follow-up is ongoing to determine whether regurgitation at 1 year will translate into a net clinical benefit for patients undergoing $\mathrm{CABG}+\mathrm{MVR}$.

\section{Ischemic Heart Disease: Drugs, Devices, and Systems of Care}

Air Versus Oxygen in ST-Elevation Myocardial Infarction (AVOID) Oxygen therapy has been standard care for MI for over a century, but the science is limited with respect to whether it is helpful when patients are not hypoxic. In the AVOID study, ST-elevation MI patients with normal oxygen levels were randomized to receive supplemental oxygen $(n=218)$ or no oxygen $(n=223)$. Supplemental oxygen therapy in patients with ST-elevation MI, but without hypoxia, increased the incidence of myocardial injury (area under curve of creatinine kinase; $\mathrm{P}=0.04)$, recurrent $\mathrm{MI}$ at hospital discharge $(\mathrm{P}<0.01)$, and major cardiac arrhythmia $(\mathrm{P}=0.05)$, and was associated with larger myocardial infarct size assessed using cardiac magnetic resonance imaging at 6 months $(\mathrm{P}=0.04)$.

BASKET-PROVE II BASKET-PROVE II was a multicenter, open-label, randomized safety and long-term efficacy study comparing 3 types of coronary stents (the durable-polymer DES [DP-DES], bare-metal stent [BMS], and biodegradablepolymer DES [BP-DES]). ${ }^{14}$ The study randomized 2,291 patients who presented with acute or stable coronary disease requiring stents $\geq 3.0 \mathrm{~mm}$ in diameter to biolimus-A9-eluting BP-DES, second-generation everolimus-eluting DP-DES, or thin-strut silicon-carbide-coated BMS. The cumulative incidence of the primary endpoint (cardiac death, MI, and targetvessel restenosis within 2 years) was $7.6 \%$ with BP-DES, $6.8 \%$ with DP-DES, and $12.7 \%$ with BMS. BP-DES was noninferior to DP-DES and superior to BMS after 2 years in a realworld population of patients in need of large-vessel stenting. These findings challenge the concept of the polymer as key to perceived late deficiency of DP-DES; however, this may only be proven via a mega-trial.

EVOLVE II EVOLVE II assesses the safety and effectiveness of the SYNERGY Everolimus-Eluting Platinum Chromium Coronary Stent System for the treatment of patients with atherosclerotic lesions in this prospective, multicenter trial. The SYNERGY stent ( $\mathrm{n}=846$ ), which combines a drug (everolimus) in a bioabsorbable polymer coating and a device (platinum chromium stent platform), proved noninferior to the Promus Element Plus stent $(\mathrm{n}=838)$ for target lesion failure at 1 year. The relative long-term efficacy and safety of the SYNERGY stent is currently under evaluation.

\section{Clinical Science Special Reports: Off the Beaten Pathologies}

"Clinical Science Special Reports: Off the Beaten Patholo- gies" was a good opportunity to obtain secondary analyses from large trials or the primary results of smaller randomized clinical trials in the field of HF. This session was organized by Professors Hiroaki Shimokawa, from Tohoku University, and JoAnn Lindenfield, from the University of Colorado. Investigators of PARADIM-HF, one of the landmark studies in the $\mathrm{CV}$ field in $2014,{ }^{15}$ presented that, relative to enalapril, the angiotensin receptor-neprilysin inhibitor LCZ696 slowed progression of HF, delaying or preventing both nonfatal and fatal HF worsening. PreSERVE investigators showed that intracoronary administration of autologous $\mathrm{CD} 34^{+}$cells was safe and associated with a statistically significant reduction in mortality in ST-elevation MI patients with reduced ejection fraction (EF). DEFEAT-HF investigators showed that spinal cord stimulation does not offer any clinical benefit or improvement in clinical outcomes as a treatment for HF. Use of excellent guideline-determined medical therapy and little remodeling in stable HF patients may have rendered the demonstration of efficacy challenging in this short-term study. The AUGMENT-HF study was presented to show the efficacy of left ventricular augmentation with Algisyl-LVR ${ }^{\mathrm{TM}}$, a proprietary alginate hydrogel that is injected into the midwall of the left ventricle and intended to reduce left ventricular wall stress and prevent or reverse the progression of HF. This study provided proof of the concept of left ventricular reconstruction with Algisyl-LVR ${ }^{\mathrm{TM}}$ as a potential novel therapy for patients with advanced HF.

\section{Special Session: State of the Art - HF, and Preserved EF}

HF with preserved EF (HFpEF) is a hot topic in CV research, and the Special Session was delivered to a full audience. Professor Shin-ichi Momomura from Jichii Medical University Saitama Medical School presented the heterogeneity of HFpEF and showed several approaches to matching key HFpEF phenotypes and comorbidities to select therapeutic interventions. He identified one of the therapeutic targets as sleep apnea, particularly with respect to the effect of continuous positive air pressure on diastolic function in patients with obstructive sleep apnea. ${ }^{16}$ Adaptive servo ventilation was also introduced as a novel therapeutic strategy in patients with $\mathrm{HFpEF}$ and Cheyne-Stoke respiration. ${ }^{17}$

\section{Closing Remarks}

Although the European Society of Cardiology has gained popularity among Japanese CV researchers, the AHA Scientific Sessions is definitely a superior event in which we can truly interact with both clinicians and basic research scientists. Despite the convenience of PubMed or Session OnDemand ${ }^{\mathrm{TM}}$ Premium, personal interaction at the Scientific Sessions is priceless and could provide memorable moments in our research lives. At times, great ideas arise from questions asked in the AHA Scientific Sessions. It is my hope that my report, although only a brief summary of the latest knowledge, will generate interest among young Japanese cardiologists to submit abstracts and attend the AHA Scientific Sessions in Orlando, Florida, between November 7 and 11, 2015.

\section{Acknowledgments}

The author thanks all the attendees of the AHA Scientific Sessions, 2014 from the Division of Cardiology, Department of Medicine, Keio University School of Medicine for their thoughtful comments. 


\section{Disclosures}

No financial support was received for this study.

\section{References}

1. Ishimori $\mathrm{N}$, Kinugawa $\mathrm{S}$, Yamada $\mathrm{S}$, Yokoshiki $\mathrm{H}$, Mitsuyama $\mathrm{H}$, Tsutsui H. Report of the American Heart Association (AHA) scientific sessions 2013, Dallas. Circ J 2014; 78: 51-56.

2. Muramatsu T, Ozaki Y. European Society of Cardiology (ESC) congress report from Barcelona 2014. Circ J 2014; 78: 2610-2618.

3. Kusunose K. Report of the American College of Cardiology (ACC) Scientific Sessions 2014, Washington, DC. Circ J 2014; 78: 1311 1316.

4. Giugliano RP, Ruff CT, Braunwald E, Murphy SA, Wiviott SD, Halperin JL, et al; ENGAGE AF-TIMI 48 Investigators. Edoxaban versus warfarin in patients with atrial fibrillation. $N$ Engl $J$ Med 2013; 369: 2093-2104.

5. Hemmi N, Tohyama S, Nakajima K, Kanazawa H, Suzuki T, Hattori $\mathrm{F}$, et al. A massive suspension culture system with metabolic purification for human pluripotent stem cell-derived cardiomyocytes. Stem Cells Transl Med 2014; 3: 1473-1483.

6. Takeda N, Manabe I, Uchino Y, Eguchi K, Matsumoto S, Nishimura $\mathrm{S}$, et al. Cardiac fibroblasts are essential for the adaptive response of the murine heart to pressure overload. J Clin Invest 2010; 120: $254-265$.

7. Mauri L, Kereiakes DJ, Yeh RW, Driscoll-Shempp P, Cutlip DE, Steg PG, et al; the DAPT Study Investigators. Twelve or 30 months of dual antiplatelet therapy after drug-eluting stents. $N$ Engl J Med 2014; 371: 2155-2166

8. Kimura T, Morimoto T, Nakagawa Y, Kawai K, Miyazaki S, Muramatsu T, et al; J-Cypher Registry Investigators. Very late stent thrombosis and late target lesion revascularization after sirolimuseluting stent implantation: Five-year outcome of the J-CYPHER registry. Circulation 2012; 125: 584-591.

9. Garratt KN, Weaver WD, Jenkins RD, Pow TK, Mauri L, Kereiakes DJ, et al. Prasugrel plus aspirin beyond 12 months is associated with improved outcomes after taxus liberte paclitaxel-eluting coronary stent placement. Circulation 2014 November 16, doi:10.1161/
CIRCULATIONAHA.114.013570.

10. Ikeda Y, Shimada K, Teramoto T, Uchiyama S, Yamazaki T, Oikawa $\mathrm{S}$, et al. Low-dose aspirin for primary prevention of cardiovascular events in japanese patients 60 years or older with atherosclerotic risk factors: A randomized clinical trial. JAMA 2014 November 17, doi:10.1001/jama.2014.15690.

11. Muhlestein JB, Lappe DL, Lima JA, Rosen BD, May HT, Knight S, et al. Effect of screening for coronary artery disease using ct angiography on mortality and cardiac events in high-risk patients with diabetes: The FACTOR-64 randomized clinical trial. JAMA 2014; 312: 2234-2243.

12. Lacro RV, Dietz HC, Sleeper LA, Yetman AT, Bradley TJ, Colan $\mathrm{SD}$, et al; the Pediatric Heart Network Investigators. Atenolol versus losartan in children and young adults with marfan's syndrome. $N$ Engl J Med 2014; 371: 2061 - 2071.

13. Smith PK, Puskas JD, Ascheim DD, Voisine P, Gelijns AC, Moskowitz AJ, et al; the Cardiothoracic Surgical Trials Network Investigators. Surgical treatment of moderate ischemic mitral regurgitation. N Engl J Med 2014; 371: 2178-2188.

14. Kaiser C, Galatius S, Jeger R, Gilgen N, Jensen JS, Naber CK, et al. Long-term efficacy and safety of biodegradable-polymer biolimuseluting stents: Main results of the Basel Stent Kosten-Effektivitats Trial- Prospective Validation Examination II (BASKET-PROVE II), a randomized, controlled noninferiority 2-year outcome trial. Circulation 2014 November 19, doi:10.1161/CIRCULATIONAHA.114. 013520 .

15. McMurray JJ, Packer M, Desai AS, Gong J, Lefkowitz MP, Rizkala AR, et al; PARADIGM-HF Investigators and Committees. Angiotensin-neprilysin inhibition versus enalapril in heart failure. $N$ Engl J Med 2014; 371: 993-1004.

16. Butt M, Dwivedi G, Shantsila A, Khair OA, Lip GY. Left ventricular systolic and diastolic function in obstructive sleep apnea: Impact of continuous positive airway pressure therapy. Circ Heart Fail 2012; 5: $226-233$.

17. Yoshihisa A, Suzuki S, Yamaki T, Sugimoto K, Kunii H, Nakazato $\mathrm{K}$, et al. Impact of adaptive servo-ventilation on cardiovascular function and prognosis in heart failure patients with preserved left ventricular ejection fraction and sleep-disordered breathing. Eur J Heart Fail 2013; 15: 543-550. 